\title{
A INSERÇÃO DO RESIDENTE EM ENFERMAGEM EM UMA UNIDADE DE INTERNAÇÃO CIRÚRGICA: PRÁTICAS E DESAFIOS*
}

\author{
Livia Crespo Drago ${ }^{1}$, Roberta Luiza Salum², Selma Regina de Andrade 3 , Michele Medeiros ${ }^{4}$, \\ Monique Mendes Marinho
}

RESUMO: A inclusão de residentes em enfermagem pode promover mudanças no ambiente do cuidado em âmbito hospitalar. Esta pesquisa objetivou compreender se houve, ou não, mudança nas práticas de enfermagem, a partir da inserção de residentes dessa categoria em uma unidade de internação de Hospital Universitário, na percepção da equipe de enfermagem. Trata-se de pesquisa exploratório-descritiva, de abordagem qualitativa, utilizando a técnica de análise de Bardin e da qual resultaram duas categorias: Perspectiva do profissional técnico de enfermagem e Perspectiva das enfermeiras. Os participantes perceberam não haver mudanças substantivas nas práticas a partir da inserção dos residentes de enfermagem. Foram identificadas situações intermediárias, entendidas como desafios ao Programa. O aperfeiçoamento dos modos de inserção do residente nos cenários de prática poderá contribuir para a educação em enfermagem, o processo de educação permanente em saúde, e para o cuidado prestado nas instituições à sociedade. DESCRITORES: Internato não médico; Enfermagem; Serviço hospitalar de enfermagem; Cuidados de enfermagem; Inovação organizacional.

\section{THE INSERTION OF THE RESIDENT IN NURSING IN A SURGICAL CARE UNIT: PRACTICES AND CHALLENGES}

\begin{abstract}
The inclusion of nursing residents can promote changes in the care environment in the hospital ambit. This research aimed to understand whether changes took place or not in the nursing practices, as a result of the insertion of residents of this category in an inpatient unit in a University Hospital, in the perception of the nursing team. This research is exploratorydescriptive, has a qualitative approach, and uses the Bardin analysis technique, from which two categories resulted: The perspective of the nurse technicians and The perspective of the nurses. The participants did not perceive substantive changes in the practices resulting from the insertion of the nursing residents. Intermediate situations were identified, which were understood as challenges to the Program. The improvement of modes of insertion of the resident in the practice settings could contribute to education in nursing, to the process of continuing education in health, and to the care given in the institutions to society.
\end{abstract}

DESCRIPTORS: Non medical internships; Nursing; Hospital nursing service; Nursing care; Organizational innovation.

\section{LA INSERCIÓN DEL RESIDENTE EN ENFERMERÍA EN UNA UNIDAD DE INTERNACIÓN QUIRÚRGICA: PRÁCTICAS Y DESAFÍOS}

RESUMEN: La inclusión de residentes en enfermería puede promover cambios en el ambiente del cuidado en ámbito hospitalar. Esta investigación tuvo el objetivo de comprender se hubo o no cambio en las prácticas de enfermería observando la inserción de residentes de esa categoría en una unidad de internación de Hospital Universitario, en la percepción del equipo de enfermería. Es una investigación exploratorio descriptiva, de abordaje cualitativo, utilizando la técnica de análisis de Bardin y de la cual resultaron dos categorías: Perspectiva del profesional técnico de enfermería y Perspectiva de las enfermeras. Los participantes percibieron no haber cambios sustantivos en las prácticas a partir de la inserción de los residentes de enfermería. Fueron identificadas situaciones intermediarias, entendidas como desafíos al Programa. El perfeccionamiento de los modos de inserción del residente en los escenarios de práctica podrá contribuir para la educación en enfermería, el proceso de educación permanente en salud, y para el cuidado prestado en las instituciones a la sociedad.

DESCRIPTORES: Internado no médico; Enfermería; Servicio hospitalar de enfermería; Cuidados de enfermería; Innovación organizacional.

${ }^{1}$ Enfermeira. Naturóloga. Mestranda em Enfermagem pelo Programa de Pós-Graduacao em Enfermagem da Universidade Federal de Santa Catarina - PEN UFSC. Bolsista CAPES pelo Projeto Pró-Ensino em Saúde. Membro do Grupo de Estudos e Pesquisa em Administração, Gerência do Cuidado e Gestão Educacional em Enfermagem e Saúde - GEPADES.

${ }^{2}$ Enfermeira.

${ }^{3}$ Enfermeira. Doutora em Enfermagem. Professora do Departamento de Enfermagem e do PEN UFSC. Vice-líder do GEPADES.

${ }^{4}$ Enfermeira do Hospital Universitário da UFSC. Especialista em Gerontologia. Membro do Grupo de Pesquisa em Tecnologias, Informações e Informática em Saúde e Enfermagem - GIATE.

${ }^{5}$ Enfermeira do Hospital Universitário da UFSC. Mestre em Enfermagem. Doutoranda pelo PEN UFSC. Membro do Grupo de Pesquisa Cuidando e Confortando - C\&C.

Autor correspondente:

Recebido: $23 / 08 / 2012$

Livia Crespo Drago

Aprovado: 20/02/2013

Universidade Federal de Santa Catarina

R. Ogê Fortkamp, 111 - 88036-610 - Florianópolis-SC-Brasil

E-mail: liviacdrago@hotmail.com 


\section{INTRODUÇÃO}

A residência é uma modalidade de pós-graduação lato sensu, caracterizada pela formação em serviço em instituições de saúde, com dedicação exclusiva, sob orientação e supervisão de profissionais capacitados. A residência multiprofissional em saúde integra a política nacional de educação e desenvolvimento do Sistema Único de Saúde (SUS), incluindo diferentes categorias profissionais, com vistas à formação coletiva, voltada à produção da atenção integral ao usuário ${ }^{(1)}$.

Com a criação de residências multiprofissionais e em área profissional da saúde, pela Lei n. 11.129 de 2005 e sua regulamentação em 2007, foi criada a Comissão Nacional de Residência Multiprofissional de Saúde (CNRMS) $)^{(1-3)}$, que cadastrou a Residência Integrada Multiprofissional em Saúde (RIMS) dos Hospitais Universitários em 2010, em fase de autorização e reconhecimento. Neste mesmo ano, teve início o Programa em Residência Integrada Multiprofissional em Saúde (RIMS) de um Hospital Universitário, com oferta de duas áreas de concentração: Atenção em Urgência e Emergência e Atenção em Alta complexidade em saúde. Dentre as profissões que se incluem na RIMS estão: enfermagem, odontologia, nutrição, farmácia, serviço social e psicologia ${ }^{(4)}$.

Uma breve revisão da literatura a respeito da residência em enfermagem mostrou que a produção sobre esta temática ainda é escassa, com abordagens diversificadas. Em geral, os estudos ressaltam a visão ou percepção dos residentes em face aos processos da pós-graduação. Entre elas, destacam-se: qualidade de vida e saúde mental dos residentes ${ }^{(5)}$; a análise de discursos sobre a Residência Multiprofissional ${ }^{(6)}$; caracterização do cuidado de enfermagem dos egressos da Residência de Enfermagem em Instituição Hospitalar Especializada em Câncer ${ }^{(7)}$; inserção dos especialistas no mercado de trabalho, em outros programas de pós-graduação e o significado da Residência para a sua vida profissional ${ }^{(8)}$; repercussão da residência nos cenários de estágios e a percepção dos residentes ${ }^{(9)}$; processo educativo e os resultados de aprendizagem na Residência de Enfermagem Materno-Infantil( ${ }^{(10)}$; e formação da Residência Multiprofissional ${ }^{(11)}$.

Uma experiência institucional de produção coletiva sobre Residências em Saúde, publicada em 2010, apresentou reflexões sobre esta modalidade de formação profissional, enfatizando a estrutura e os processos da especialização, os serviços como espaços dessa formação e a relevância da qualificação profissional comprometida com os princípios do SUS ${ }^{(12)}$.

Embora se trate de uma modalidade de formação profissional integrada, recentemente implementada, alguns questionamentos são feitos sobre as possibilidades de mudanças nas práticas assistenciais desde o ingresso do residente. Neste sentido, a introdução da RIMS pode ser caracterizada como uma mudança organizacional, entendida como toda e qualquer alteração de natureza estrutural, estratégica, cultural, tecnológica, humana ou de outro componente, capaz de provocar impacto em partes ou em toda a organização ${ }^{(13)}$.

A mudança organizacional algumas vezes é percebida como uma atitude reativa a um fenômeno que ocorre, sendo uma resposta a estímulos, tanto internos, como externos $^{(14)}$. Muitos são os aspectos que podem ser considerados para definir mudança. No entanto, suas principais características incluem a intensidade, $o$ tempo de reação e as pessoas envolvidas. Atualmente, a maioria das organizações de saúde passam por mudanças contínuas, podendo estar voltadas à reestruturação organizacional e/ou para a melhoria de qualidade ${ }^{(15)}$. $\mathrm{Na}$ ocorrência de uma mudança organizacional, as reações mais frequentemente encontradas são de resistência, expectativa ou ameaça ${ }^{(16)}$.

A pergunta de pesquisa que norteou o presente estudo teve a seguinte proposição: Quais foram as mudanças das práticas de enfermagem, sob a perspectiva da equipe de enfermagem de uma unidade de internação cirúrgica, a partir da inserção da residência em enfermagem? Compreender as possíveis alterações nas práticas de assistência ou de gerência do cuidado em enfermagem, na perspectiva dos trabalhadores de saúde, a partir da iniciativa de inclusão de residentes dessa categoria profissional, constituiu o objetivo desta pesquisa.

\section{MÉTODO}

Pesquisa exploratória e descritiva, de natureza qualitativa, realizada em uma unidade de internação cirúrgica de um hospital universitário. A coleta de dados ocorreu no mês de abril de 2011. Foram considerados os seguintes critérios de inclusão para seleção dos entrevistados: ter contato com o trabalho das residentes de enfermagem; ser membro da equipe desde, no mínimo, setembro de 2009; trabalhar no período diurno; estar no exercício da sua função. Foram entrevistados, individualmente, todos os técnicos em enfermagem e enfermeiros que atenderam aos critérios de inclusão e que consentiram em participar da pesquisa, nos termos da Resolução 196/96 do Conselho Nacional de Saúde. 
As entrevistas foram gravadas e depois transcritas e seguiram um roteiro semiestruturado, com duração aproximada de 20 minutos. Foram realizadas no local do estudo, em horário agendado pelos participantes.

A análise foi efetuada através da categorização dos dados, codificando e representando as impressões obtidas $^{(17)}$. A interpretação, a partir da categorização, proporcionou desenvolver as explicações, proposições e afirmativas necessárias para o alcance do objetivo proposto. Da análise resultaram duas categorias: Perspectiva do profissional técnico em enfermagem e Perspectiva das enfermeiras.

A realização desta pesquisa seguiu as normas do Conselho Nacional de Saúde e da Comissão Nacional de Ética em Pesquisa, obtendo aprovação do Comitê de Ética em Pesquisa com Seres Humanos, certificada pelo parecer n. 1618/11. Foi garantido aos participantes da pesquisa o sigilo e a condencialidade dos dados.

\section{RESULTADOS}

A implantação da RIMS e a inserção do residente em enfermagem nas unidades de internação de um hospital universitário levaram à compreensão das práticas e dos desafios tanto com relação à formação profissional, quanto sobre as alterações no cotidiano da equipe na unidade de internação cirúrgica. Na unidade de internação em estudo, a equipe de enfermagem era composta por 34 profissionais, entre enfermeiros, técnicos e auxiliares de enfermagem. Os resultados alcançados consideraram as entrevistas de todos os integrantes da equipe de enfermagem da unidade que atenderam aos critérios de inclusão e consentiram em sua participação. Sendo assim, foram entrevistados cinco técnicas em enfermagem com faixa etária entre 24 e 54 anos e tempo médio de trabalho no hospital de 9 anos e seis meses; e três enfermeiras com faixa etária variando de 25 a 35 anos e tempo médio de trabalho no hospital de 6 anos e 9 meses, totalizando 8 participantes.

Estes resultados são apresentados a partir da percepção da equipe de enfermagem, técnicos e enfermeiros, assistenciais e gerencial, organizados nas duas categorias apresentadas a seguir.

\section{Perspectiva do profissional técnico em enfermagem}

A presença e atuação das residentes de enfermagem foram observadas pela maioria dos técnicos em enfermagem entrevistados. No entanto, a observação residiu no aspecto gerencial da prática da enfermeira, mencionada como de âmbito burocrático do trabalho, ou seja, no exercício da aplicação da metodologia da assistência do cuidado. No hospital em estudo, o processo de enfermagem é composto por histórico de enfermagem, diagnóstico de enfermagem, plano assistencial, prescrição de enfermagem, evolução e prognóstico. A atuação das residentes no acompanhamento da enfermeira também foi relatada como sendo mais uma ajuda para o setor e para as enfermeiras assistenciais.

O desconhecimento do papel das residentes de enfermagem na unidade de internação cirúrgica foi relatado pelos entrevistados e a falta de sensibilização sobre a residência multiprofissional é um problema presente nos relatos da maioria dos participantes, como explicitado na fala de P1:

Os residentes de enfermagem entraram aqui, mas não foi, pelo menos para mim, colocado nada a respeito do que eles vão fazer aqui dentro. Qual é o papel deles aqui dentro, enquanto eles tiverem nosso ambiente de trabalho? Eu não sei. (P1)

Observou-se, também, certa confusão de alguns dos técnicos em enfermagem a respeito do papel das residentes e das acadêmicas de oitava fase do curso de graduação. Quando questionados sobre quais atividades eram exercidas pelas residentes de enfermagem esses, houve unanimidade nas respostas, citando os curativos complexos, os acessos profundos, as orientações aos pacientes, as evoluções diárias e as trocas de nutrição parenteral.

Mais uma vez, a referência de atuação semelhante entre as acadêmicas de oitava fase do curso de graduação e as residentes foram consideradas as mesmas. A personalidade de cada residente influência este aspecto, como podemos perceber no relato:

O setor é muito rico em aprendizado, porém não percebo as residentes aproveitando este espaço. O interesse é individual em qualquer profissão, não é diferente na enfermagem, umas residentes são mais interessadas e outra não. É complicado. (P1)

As percepções sobre as possíveis mudanças das práticas de enfermagem após a inserção do residente foram convergentes no sentido de haver mais um profissional 'ajudando' na unidade. No entanto, parte dos profissionais técnicos entrevistados não observou mudança ou contribuição do residente de enfermagem nas práticas de cuidado, já que para eles não estava claro 
o objetivo da residência e, portanto, não consideraram possível avaliar:

Mudança, eu não me recordo de nada. Eu acho que entraram na rotina e entraram prá ajudar. Eu acho que foi isso. De mudança, não me recordo. (P4)

A maioria dos profissionais técnicos percebeu dificuldades iniciais de adaptação das residentes, como inexperiência prática, desconhecimento do local de atuação e pouco contato com os profissionais que trabalham no setor. Uma dificuldade relevante relatada está relacionada ao pouco tempo que as residentes permanecem na Unidade:

Elas têm bastante dificuldade atése entrosar, e quando elas tão quase saindo é que parece que está ficando bom. (P2)

\section{Perspectiva das enfermeiras}

As enfermeiras entrevistadas têm funções diferenciadas na unidade: duas atuam diretamente na assistência e uma concentra funções gerenciais. O tempo de formatura e de trabalho na unidade entre estas enfermeiras também diferem, bem como as percepções sobre a atuação das residentes na unidade. Contudo, as enfermeiras consideram a RIMS, na área profissional de enfermagem, uma experiência muito nova, dificultando uma avaliação abrangente tanto da presença quanto da atuação das residentes de enfermagem na unidade.

As enfermeiras referem que, além de ajudar na assistência, a residência na área da enfermagem é positiva, valoriza a profissão, e é considerada uma oportunidade de aprender na prática e tornar o profissional mais preparado para o mercado.

As participantes relatam que o cronograma das residentes é descontínuo: em apenas 9 semanas as residentes passam pela unidade e em mais dois setores (centro cirúrgico e unidade de endoscopia), além das aulas teóricas, restando pouco tempo de atuação no setor. Como consequência, percebem comprometimento no desempenho das atividades. Consideram que as residentes têm certa dificuldade em acompanhar as rotinas da unidade, em criar vínculo com os pacientes e com os funcionários, e em desenvolver atividades inerentes à função no serviço. Além disso, a presença da enfermeira assistencial, responsável pelo turno, concomitante à presença do residente, parece gerar barreira ou inibição na atução do profissional em formação.
A falta autonomia das residentes de enfermagem, e sua dependência de uma supervisão, revela certo dilema: é uma enfermeira (uma vez que é graduada) ou é uma aluna (já que está em processo de formação especializada). Esta situação ambígua revela as dificuldades percebidas pelas enfermeiras a respeito da inserção das residentes. A questão que se coloca é a de ter clareza do papel da residente na unidade de internação, uma vez que não se trata de enfermeira da unidade do hospital, concursada e contratada, mas também não se trata de aluna da graduação, mas de enfermeira.

As atividades realizadas pelas residentes de enfermagem são a realização da metodologia da assistência do cuidado e a assistência direta ao paciente (tais como, curativos complexos, sondagens, gasometria). As atividades gerenciais não têm sido realizadas pelas residentes, como justifica uma enfermeira:

Até pelo fato de ela ficar pouco na unidade, ela acaba ela ficando mais na parte assistencial. Porque, se aparece uma gasometria, aparece uma sondagem, ela acaba fazendo esses procedimentos mais complexos. $E$ evolução, prescrição e essas coisas [...]. (P6)

Uma das profissionais destaca a contribuição da residente no grupo multiprofissional sobre orientações que a unidade está implantando e outra ressalta o aproveitamento desse profissional na unidade, respectivamente:

A gente pode fazer essa barganha do residente vir aprender com o setor e a gente poder ampliar e melhorar um pouco mais o serviço e a assistência. (P8)

[...] se elas ficassem aqui direto, elas aproveitariam mais, e a própria unidade poderia aproveitar mais este profissional. (P6)

Nenhuma das profissionais soube precisar uma mudança substancial nas atividades da unidade, ocorrida com a inserção da residência. No entanto, a maioria das enfermeiras afirma que a principal contribuição das residentes está em ajudar na assistência, mas que a residência em enfermagem pouco contribuiu com inovação no cuidado de enfermagem.

As enfermeiras sugerem mudanças no cronograma da RIMS para que se concentrem as atividades das residentes em um determinado setor, com vistas ao melhor aproveitamento, tanto para as residentes, quanto para a unidade. 


\section{DISCUSSÃO}

A implantação da RIMS é uma experiência recente no âmbito do Hospital Universitário. O ensino e a pesquisa em saúde são importantes aspectos para a formação profissional, produção e socialização do conhecimento, e exigem articulação mutilprofissional. Este estudo, mais do que avaliar precocemente a RIMS, buscou revelar elementos da área profissional da enfermagem, analisando possíveis relações de interdependência e de complementaridade na inserção das atividades do residente, na perspetiva da equipe de enfermagem.

Tanto enfermeiras, quanto técnicos em enfermagem consideram precoce uma avaliação da RIMS, justamente por ser uma experiência nova, iniciada em 2010 e em sua terceira turma de formação. Processos de mudança organizacional nem sempre são percebidos de imediato, visto que dependem da intensidade, do tempo de reação e das pessoas envolvidas, mas podem ser acompanhados desde seu início para oferecer rápida resposta, ou ajuste, caso surjam resistências, ou mesmo para enfrentar possíveis reações ${ }^{(14,16)}$.

As atividades exercidas pelas residentes foram observadas de igual forma pelos dois grupos profissionais, que destacaram a metodologia da assistência, atenção direta ao paciente em casos de procedimentos complexos e a ajuda da residente às ações de enfermagem na unidade. As práticas gerenciais não são realizadas pela residente devido à curta permanência na unidade durante a semana. Entretanto, espera-se que o enfermeiro, seja ele residente ou não, tenha competências gerenciais em sua prática assistencial, tais como: relacionamento interpessoal, liderança, competência técnica, tomada de decisão e compromisso ${ }^{(18)}$. De certa forma, as residentes estão desenvolvendo essas competências, mesmo que ainda não visível aos olhos da equipe.

As práticas de enfermagem vão além de cuidar e propiciar conforto aos pacientes e familiares, o enfermeiro deve ampliar este papel de modo a enfatizar a promoção da saúde e prevenção de doenças, educar, gerenciar e pesquisar ${ }^{(19)}$. Para o enfermeiro ampliar o seu papel é preciso compreender que o conhecimento, a prática e os elementos que constituem as práticas (normas e protocolos, entre outros) são indissociáveis ${ }^{(20)}$.

As atividades exercidas pelas residentes podem, em muitos casos, parecer semelhantes às desenvolvidas pelas graduandas em estágio curricular supervisionado. A confusão entre estes papéis pode ter ocorrido por falta de sensibilização com os técnicos de enfermagem sobre a função, objetivo e importância do profissional residente. É necessário fortalecer as relações entre profissionais, alunos de graduação e pós-graduação, de forma a socializar o conhecimento e ampliar a teoria e a prática, possibilitando a práxis na profissão como um dos objetivos da RIMS. Dessa maneira, o residente, ao mesmo tempo que é estudande, pode ser co-partícipe no processo de aprendizado dos graduandos, motivando esses a serem atores ativos do seu aprendizado, relacionando problemas da assistência com a literatura e, assim, desenvolvendo a competência de educador ${ }^{(21)}$.

As dificuldades foram, de certa forma, reconhecidas igualmente nos dois grupos: a insegurança e o pouco tempo na unidade. O cronograma descontínuo, a falta de autonomia, a dependência do enfermeiro do setor, fraco empoderamento para a gestão do cuidado de enfermagem e da unidade de internação são componentes que merecem discussão entre a coordenação da RIMS e os profissionais de saúde. As enfermeiras compreendem que se trata de algo novo e que este é um caminho para um melhor fazer. Por isso, sugerem alterações, principalmente, nos aspectos relacionados ao cronograma de trabalho.

O conceito de mudança organizacional possui heterogeneidade de definições. Muitos são os aspectos e características a serem considerados quando se busca conceituar mudança organizacional, dentre eles a intensidade, o tempo de reação e as pessoas envolvidas ${ }^{(14)}$. É caracterizada como qualquer alteração, planejada ou não, ocorrida nos componentes organizacionais (pessoas, trabalho, estrutura formal, cultura) ou nas relações entre a organização e seu ambiente, decorrente de fatores internos e/ou externos e que traz algum impacto nos resultados e/ou nas relações entre as pessoas ${ }^{(16)}$.

A mudança organizacional, algumas vezes, é percebida como uma atitude reativa a um fenômeno que ocorre, sendo uma resposta a estímulos, tanto internos como externos ${ }^{(14)}$. É importante ressaltar, que as mudanças não devem ser vistas como ameaça, mas como desafio, onde a possibilidade de fazer algo novo e inovador seja o incentivo necessário para todos que os envolvidos possam ser capazes de passar por este processo de forma positiva ${ }^{(15)}$.

Embora mudanças significativas nas práticas assistenciais e/ou gerenciais de enfermagem na unidade estudada, a partir da inserção do residente de enfermagem, não tenham sido observadas, a importância da residência na área de concentração e os benefícios que acarretam para a categoria foram destacados como pontos relevantes e formalizam a conquista de mais um espaço de conhecimento e prática na profissão. 
A visão de que o residente de enfermagem é 'alguém que ajuda' ou 'uma ajuda a mais' pode ser considerada temporária, visto o curto período de implementação da RIMS. Ela mesma é, em si, uma mudança substantiva nas práticas de formação em saúde, ainda em processo.

Apesar de uma mudança organizacional ter ocorrido na unidade de internação estudada, os profissionais não a identificaram como tal. A atitude reativa ${ }^{(14)}$ ocorre de maneira indireta, já que a presença das residentes é observada e há interação com a equipe. Talvez o fato de se tratar de um hospital escola, com um constante ir e vir de estudantes, seja um dos fatores para que a mudança organizacional não tenha sido percebida.

\section{CONSIDERAÇÕES FINAIS}

O estudo possibilitou identificar as atribuições assistenciais desenvolvidas pelo residente de enfermagem e analisar as contribuições e dificuldades a partir da inserção desta nova modalidade de especialização.

O desafio da mudança ainda é latente na unidade em questão, uma vez que o tempo de implantação é relativamente curto. Além dos fatores que caracterizam a mudança (intensidade, tempo de reação e pessoas envolvidas), o fato de tratar-se de um hospital de ensino pode contribuir, em parte, para que esta mudança passe despercebida pelos funcionários, visto que a rotatividade de estudantes, incluindo residentes, é muito grande.

A percepção da equipe de enfermagem de que não há mudanças substantivas nas práticas de enfermagem, a partir da inserção dos residentes, não impediram perceber alterações no cotidiano, tais como situações ainda a serem resolvidas, que se expressam como desafios ao Programa RIMS. A falta de autonomia do residente de enfermagem para práticas gerenciais, inclusive a gerência do cuidado, sua dependência pelo enfermeiro supervisor, seu papel ambíguo - profissional/aluno, o desconhecimento do papel do residente por parte da equipe de enfermagem, a falta de sensibilização dos profissionais quanto à residência, o cronograma descontínuo de atividades de formação no campo de prática e a pouca permanência no setor são fatores que dificultam uma atuação propositiva e marcante no setor. Evidencia-se a necessidade de esclarecimento às equipes que irão receber esse profissional quanto ao seu papel e seu objetivo na unidade.

O aperfeiçoamento dos modos de inserção do residente de enfermagem nos cenários de prática profissional poderá contribuir, não só para a otimização do processo de educação permanente em saúde em desenvolvimento no país mas, sobretudo, para a melhoria do cuidado prestado nas instituições. Certamente, o impacto da formação profissional obtida, na RIMS, ao longo do tempo, trará repercussões tanto para a educação em enfermagem, quanto para a sociedade que receberá os cuidados qualificados e diferenciados.

\section{REFERÊNCIAS}

1. Ministério da Educação (BR). Portaria Interministerial n. 45, de 12 de janeiro de 2007: dispõe sobre a Residência Multiprofissional em Saúde e a Residência em Área Profissional da Saúde e institui a Comissão Nacional de Residência Multiprofissional em Saúde. [Internet] Brasília (DF), 2007. [acesso em 01 nov 2011]. Disponível: http://portal.mec.gov.br/index. php?option $=$ com_docman \&task $=$ doc_view\&gid $=358$ $\& \mathrm{tmpl}=$ component\&format $=$ raw\&Itemid.

2. Brasil. Lei n. 11.129, de 30 de junho de 2005: Institui o Programa Nacional de Inclusão de Jovens - ProJovem; cria o Conselho Nacional da Juventude - CNJ e a Secretaria Nacional de Juventude; altera as Leis nos 10.683 de 28 de maio de 2003 e 10.429 de 24 de abril de 2002. [Internet] Brasília (DF), 2005. Brasília (DF), 2007. [acesso em 01 nov 2011]. Disponível: https:// wwwl.defesa.gov.br/saude/L11129.pdf

3. Brasil. Residências em Saúde. Residência Multiprofissional. Relação de Programas de Residência Multiprofissional e em Área Profissional da Saúde aguardando avaliação pela CNRMS. [Internet] 2009. [acesso em 01 nov 2011]. Disponível: http://portal.mec. gov.br/index.php?option $=$ com_content\&view $=$ article $\&$ id $=12501 \&$ Itemid $=813$

4. Andrade JS, Vieira MJ. Prática assistencial de enfermagem: problemas, perspectivas e necessidades de sistematização. Rev Bras Enferm. [Internet] 2005;58(3) [acesso em 01 nov 2011]. Disponível: http://www.scielo. br/pdf/reben/v58n3/a02v58n3.pdf

5. Franco GP, Barros ALBL, Nogueira-Martins LA. Qualidade de vida e sintomas depressivos em residentes de enfermagem. Rev. Latino-Am. Enfermagem [Internet] 2005;13(2). [acesso em 01 nov 2011]. Disponível: http:// www.scielo.br/scielo.php?script=sci_arttext\&pid=S0104$11692005000200002 \& \operatorname{lng}=\mathrm{en} \& \mathrm{nrm}=\mathrm{iso}$

6. Ferreira SR. Residência integrada em saúde: uma modalidade de ensino em serviço. Porto Alegre (RS): Universidade Federal do Rio Grande do Sul; 2007. [Internet]. [acesso em 01 nov 2011]. Disponível: http:// pesquisa.bvsalud.org/regional/resources/lil-551329 
7. Santana CJM, Lopes GT. O cuidado especializado do egresso da residência em enfermagem do Instituto Nacional de Câncer - INCA. Esc. Anna Nery. [Internet] 2007;11(3) [acesso em 01 nov 2011]. Disponível: http:// www.scielo.br/scielo.php?script=sci_arttext\&pid=S1414$81452007000300004 \& \operatorname{lng}=$ en\&nrm $=$ iso

8. Santos VP, Whitaker IY, Zanei SSV. Especialização em enfermagem modalidade residência em unidade de terapia intensiva: egressos no mercado de trabalho. Rev Gaucha Enferm. [Internet] 2007;28(2) [acesso em 01 nov 2011]. Disponível: http://pesquisa.bvsalud.org/ regional/resources/lil-534911

9. Carbogim FC, Santos KB, Alves MS, Silva GA. Residência em enfermagem: a experiência de Juiz de Fora do ponto de vista dos residentes. Rev. APS. [Internet] 2010;13(2) [acesso em 01 nov 2011]. Disponível: http://www.aps.ufjf.br/index.php/aps/ article/download/616/321

10. Bocalandro OL. Resultados en la formación del primer grupo de residentes de la especialidad en enfermería materno infantil. Rev Cubana Enfermer. [Internet] 2009;25(1/2) [acesso em 01 nov 2011]. Disponível: http://pesquisa.bvsalud.org/regional/ resources/lil-547067

11. Dallegrave D, Kruse MHL. No olho do furacão, na ilha da fantasia: a invenção da residência multiprofissional em saúde. Interface - Comunic. Saude Educ. [Internet] 2009;13(28) [acesso em 01 nov 2011]. Disponível: http://www.scielo. $\mathrm{br} / \mathrm{scielo}$.php?script $=$ sci_arttext\&pid=S1414$32832009000100018 \& \operatorname{lng}=$ en $\& n r m=$ iso

12. Brasil. Decreto n. 7.082 de 27 de janeiro de 2010: dispões sobre o Programa Nacional de Reestruturação dos Hospitais Universitários Federais - REHUF. [acesso em 01 nov 2011]. Disponível: http://www.planalto.gov.br/ ccivil_03/_Ato2007-2010/2010/Decreto/D7082.htm.

13. Wood Júnior T (Coord.). Mudança organizacional. $5^{\text {a }}$ ed. São Paulo: Atlas; 2009.

14. Sekyia SR, Luz TR. Mudança organizacional: implantação da iniciativa Hospital Amigo da Criança. Ciênc. saúde colet. [Internet] 2010;15(supl. 1) [acesso em 01 nov 2011]. Disponível: http://www.scielosp.org/scielo. php? pid $=$ S1413-81232010000700035\&script $=$ sci arttext.

15. Marquis BL, Huston CJ. Administração e liderança em enfermagem: teoria e prática. $6^{\mathrm{a}}$ ed. Porto Alegre: Artmed; 2010.
16. Bressan CL. Mudança organizacional: uma visão gerencial. Seminário de Gestão de Negócios. Curitiba: FAE; 2004. [Internet] [acesso em 01 nov 2011]. Disponível: www.fae.edu/publicacoes/pdf/art_cie/ art_25.pdf.

17. Bardin L. Análise de conteúdo. Lisboa: Edições 70; 2004.

18. Lelli L, Bernardinho E, Peres A, Fabriz L. Estratégias gerenciais para o desenvolvimento de competências em enfermagem em hospital de ensino. Cogitare enferm. [Internet] 2012;17(2) [acesso em 01 jul 2012]. Disponível: http://ojs.c3sl.ufpr.br/ojs2/index.php/ cogitare/article/view/24932/18480.

19. Potter PA, Perry AG. Fundamentos de enfermagem. Rio de Janeiro: Elsevier; 2005.

20. Velloso ISC, Ceci C, Alves M. Reflexões sobre relações de poder na prática de enfermagem. Rev. Gaúcha Enferm. [Internet] 2010;31(2) [acesso em 01 nov 2011]. Disponível: http://www.scielo.br/pdf/rgenf/v31n2/26.pdf

21. Vannuchi MTO, Campos JJB. A metodologia ativa na residência em gerência do curso de enfermagem da UEL. Cogitare enferm. [Internet] 2007;12(3) [acesso em 01 jul 2012]. Disponível: http://ojs.c3sl.ufpr.br/ojs2/ index.php/cogitare/article/view/10034/6892 